Research Paper

\title{
Peri-0perative Treatment of Giant Nodular Goiter
}

\section{Bo Gao*, Wuguo Tian*, Yan Jiang, Xiaohua Zhang, Jianjie Zhao, Shu Zhang, Jinping Chen, Donglin Luo ${ }^{\bowtie}$}

Department of Surgery for Breast and Thyroid, Institute of Surgery Research, Daping Hospital, Third Military Medical University, Chongqing 400042, China.

* Bo Gao and Wuguo Tian contribute to this study equally.

$\triangle$ Corresponding author: Dr. Donglin Luo, Department of Surgery for Breast and Thyroid, Institute of Surgery Research, Daping Hospital, Third Military Medical University, Chongqing 400042, China; Tel: +86-023-68811229; Email: luodonglin@dphospital.tmmu.edu.cn; luo32432@sina.com.

(c) Ivyspring International Publisher. This is an open-access article distributed under the terms of the Creative Commons License (http://creativecommons.org/ licenses/by-nc-nd/3.0/). Reproduction is permitted for personal, noncommercial use, provided that the article is in whole, unmodified, and properly cited.

Received: 2012.08.29; Accepted: 2012.10.15; Published: 2012.10.24

\begin{abstract}
Objective: To summarize the experience in the peri-operative treatment of giant nodular goiter.

Methods: A total of 123 patients with giant nodular goiter sized $6 \sim 20 \mathrm{~cm}$ were admitted into our hospital from 1990 to $201 \mathrm{I}$ and the clinical data were retrospectively analyzed. These patients underwent total or subtotal thyroidectomy.

Results: All patients underwent surgical intervention. Unilateral subtotal thyroidectomy was performed in 40 patients, unilateral total thyroidectomy in I patient, bilateral subtotal thyroidectomy in 79 patients, and unilateral total thyroidectomy, removal of entire isthmus and contralateral subtotal thyroidectomy in 3 patients. Nodular goiter was pathologically proven post-operatively. No short-term complications such as dyspnea or thyroid storm were found postoperatively. Post-operative follow up was done for 9 months to 6 years and no recurrence was observed.

Conclusion: Comprehensive pre-operative preparation, pre-operative evaluation, complete exposure of the operative field, meticulous operation, effective control and prevention of hemorrhage and prevention against damage to superior and recurrent laryngeal nerves are crucial for the successful surgical intervention of giant nodular goiter.
\end{abstract}

Key words: nodular goiter; peri-operative treatment; surgical intervention.

\section{Introduction}

Nodular goiter is the most common benign disease of the thyroid and can be found in any age group. The incidence of nodular goiter was $5.0 \sim 10.0 \%$ and it was 4 times higher in females than that in males. The incidence of nodular goiter is at a higher level, and related studies reveal the thyroid nodules are found in $4.0 \sim 8.0 \%$ of adults at palpation and the incidence of thyroid nodular disease is $30 \sim 50 \%$ in general population at ultrasonography [1-4]. At the early stage of nodular goiter, the nodule is small and the compression symptoms are not evident. Thus, it is difficult to diagnose, and confirmed diagnosis is usually delayed. When the nodules are large enough or the compression symptoms become obvious, giant nodular goiter is life-threatening. To date, the general guideline for the diagnosis and treatment of nodular goiter has not developed internationally [5]. The therapeutic strategies for nodular goiter include thyroid hormone replacement therapy, radioactive Iodine I-131 therapy and surgical intervention. The thyroid hormone replacement therapy is simple and easy to perform, but the therapeutic efficacy is not satisfactory [6]. Huys- 
mans et al performed radioactive Iodine I-131 therapy in patients with nodular goiter achieving favorable therapeutic effectiveness [7]. However, some physicians postulate that radioactive Iodine I-131 therapy might have the risk for permanent thyroid dysfunction or recurrence of nodular goiter. Thus, the majority of patients with nodular goiter received surgical intervention in clinical practice [8-9]. However, when the nodules are giant, improper peri-operative treatment might cause some complications such as thyroid storm, laryngeal edema, massive hemorrhage and damage to the nerves, trachea, esophagus and parathyroid. In addition, long-lasting compression of the trachea and esophagus may cause tracheomalacia, which may cause post-operative airway collapse. Moreover, substernal goiter is still a challenge for surgeons in the Department of Thyroid Surgery. In the present study, we retrospectively reviewed 123 patients with giant nodular goiter $(6 \sim 20 \mathrm{~cm})$ who were admitted into our hospital from 1990 to 2011. The general information and findings in imaging examination were reviewed and the experience of peri-operative treatment of these patients was summarized.

\section{Materials and methods}

\section{General information}

A total of 123 patients were admitted into the Department of General Surgery from 1990 to 2011 and the nodule size ranges from $6 \mathrm{~cm}$ to $20 \mathrm{~cm}$. There were 53 males and 70 females with the male to female ratio of $1: 1.32$. The mean age was $46.5 \pm 29.5$. Unilateral goiter was found in 42 patients and bilateral goiters in 81 patients. In addition, concomitant hyperthyroidism was noted in 15 patients and thyroid cancer in 3 (Figure 1).
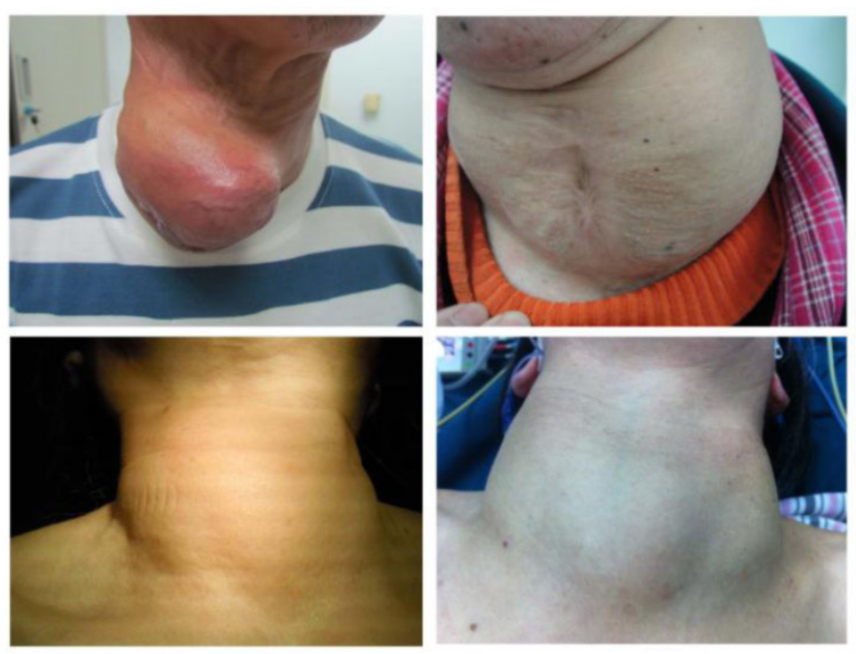

\section{Clinical manifestations}

Patients were admitted due to cervical mass. Ultrasonography showed thyroid nodules measured 6 20 cm. In several patients, the upper thyroid reached the mandibular angle, the lower part extended to the retrosternal region and the side thyroid was found at the back edge of sternocleidomastoid. Of these patients, 46 had dysphagia or neck discomfort, 25 developed tracheal compression symptoms (cough, dyspnea or hypoxemia).

\section{Laboratory and electrocardiographic exami- nation}

FT3, FT4 and TSH were measured in all patients. Results showed increase TSH in 30 patients and increased FT3 and FT4 in 15 patients. The remaining patients had normal FT3, FT4 and TSH, and routine blood test showed normal. Electrocardiography showed occasional premature ventricular contraction in 2 patients, occasional premature atrial contraction in 4 and myocardial ischemia in 5 patients.

\section{Imaging examination}

Ultrasonography and CT were employed to detect the location, size, number, border and density of nodules, and determine the tracheal compression, dislocation or stenosis. In the present study, results showed the enlargement of the thyroid which had nodules with various densities. Single nodule was found in 27 patients and multiple nodules accompanied by calcification in 5 patients. The nodules of $6 \sim 10$ $\mathrm{cm}$ in size were found in 96 patients and those of $>10$ $\mathrm{cm}$ in 27. In addition, the nodules had clear border in 77 patients and unclear border in 34 . Complete capsule of the thyroid was found in 12 patients. Annular calcification was noted in 15 patients and punctate calcification in 12 patients (Figure 2).

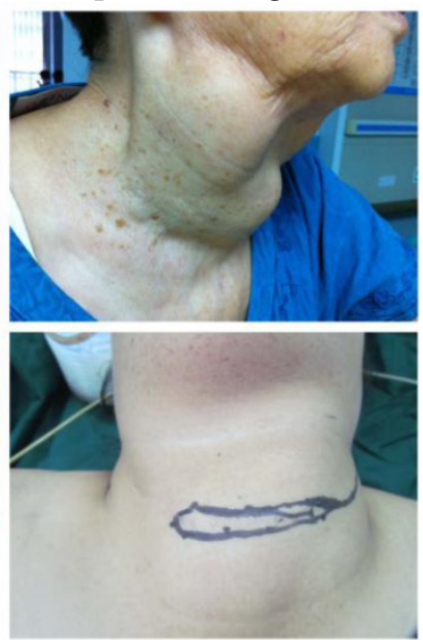

Figure I Giant mass in the neck. 

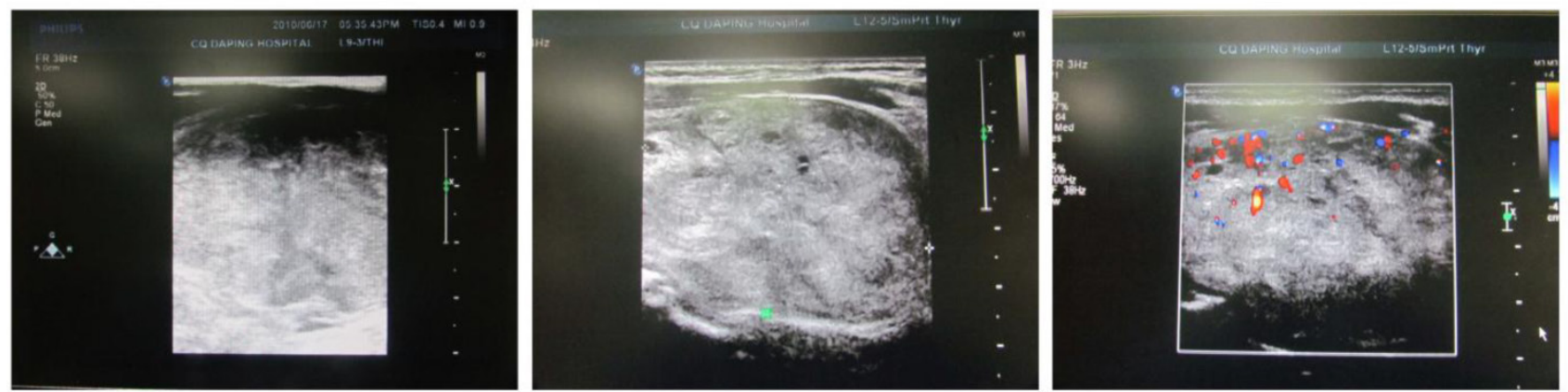

Figure 2 Ultrasonography of giant nodular goiter. Hypoechoic nodules with clear border were found. CDFI: blood flow signal was noted in the nodules.
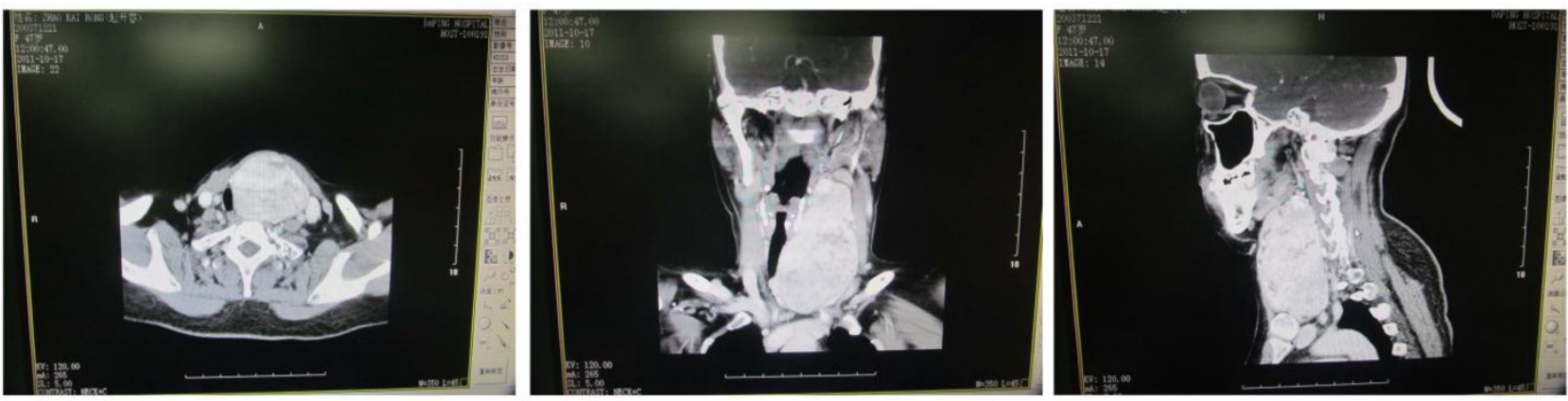

Figure 3 CT of giant nodular goiter. The thyroid was enlarged and uneven enhancement was noted. Surrounding organs were pushed toward right side.
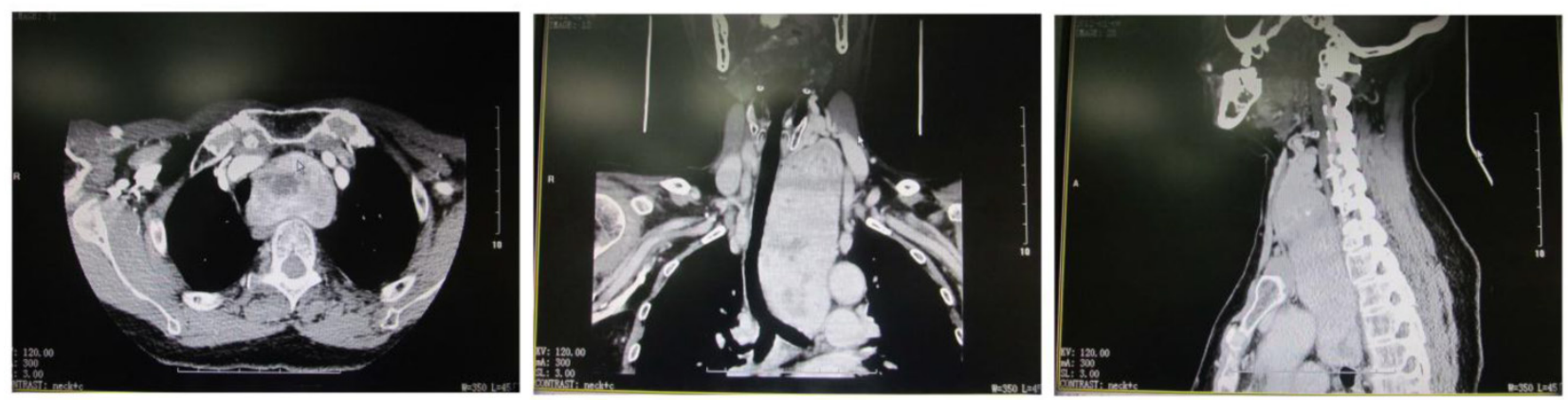

Figure 4 CT of retrosternal giant nodular goiter. The oval mass was found between the left neck and the Tracheal bifurcation and had clear border but uneven density. Uneven enhancement was noted. The surrounding tissues were compressed and the trachea was pushed toward the right side.

Plain CT scan showed the lesion was focal and enhancement CT scan revealed enhancement of different extents. There were 46 patients with esophageal compression and 25 had tracheal compression (Figure 3). Cervical $X$ ray can reveal the tracheal compression, dislocation or stenosis. Positive lateral $\mathrm{X}$ ray can reveal the degree of tracheal compression. The difference in tracheal diameter between inspiration and expiration of $>5 \mathrm{~mm}$ can be used to diagnose tracheomalacia. Retrosternal giant goiter was manifested by mass with different densities in the mediastinal region, and enhancement CT showed uneven enhancement accompanied by compression of adjacent organs (Figure 4).

\section{Laryngoscopy}

Laryngoscopy showed the location and movement of bilateral vocal cords. There were no significant abnormalities in the location and movement of the vocal cords in the 123 patients.

\section{Fine needle aspiration cytology}

For patients with clearly located nodules, fine needle aspiration was done. Cytological examination showed papillary thyroid carcinoma in 2 patients and the remaining patients were diagnosed with nodular 
goiter. Intra-operative pathology revealed papillary thyroid carcinoma in 3 patients and the remaining 120 patients had benign lesions. Post-operative pathology indicated nodular goiter in 120 patients and papillary thyroid carcinoma in 3 patients.

\section{Surgical procedures}

After comprehensive pre-operative preparation, surgery was performed. The skin and subcutaneous tissues were separated and electrocoagulation was employed for hemostasis. An incision was made between the platysma and deep cervical fascia and the thyroid capsule was dissected at the neck white line. The muscles before the thyroid received blunt dissection and the thyroid was completely exposed. If the bilateral lobes of the thyroid were not entirely exposed, partial or complete disconnection was performed at the muscles before the thyroid and sternocleidomastoid. The thyroid was explored, and total or subtotal thyroidectomy was performed depending on the nature (benign or malignant), number, distribution and pathology of the nodules. During surgery, bleeding was stopped with ultrasound knife and silk suture. In subtotal thyroidectomy, a little of normal dorsal thyroid tissues (around 5\% of the whole gland in volume) was preserved.

\section{Results}

\section{Intra-operative findings}

All patients underwent surgical intervention. Unilateral subtotal thyroidectomy was done in 40 patients, unilateral total thyroidectomy in 1 and bilateral subtotal thyroidectomy in 79 patients (Table 1 ). For 3 patients with papillary thyroid carcinoma, the affected thyroid and thyroid isthmus were completely removed and contralateral subtotal thyroidectomy and cervical lymph node dissection were also done (Figure 5). In 1 patient with goiter at the posterior mediastinum, an incision was made at the neck and chest and total thyroidectomy was performed in the affected thyroid (Figure 6).

\section{Post-operative pathological examination}

Post-operative pathology revealed nodular goiter in 120 patients and 3 patients had concomitant papillary thyroid carcinoma (Figure 7).

Table I. The percentage of various surgical procedures

\begin{tabular}{llll}
\hline Surgical procedures & Number of patients & Total & The percentage (\%) \\
\hline Unilateral subtotal thyroidectomy & 40 & 123 & 32.5 \\
$\begin{array}{l}\text { Bilateral subtotal thyroidectomy } \\
\begin{array}{l}\text { Total resection of the diseased thyroid gland and isthemus, subtotal } \\
\text { thyroidectomy and diseased cervical lymph node dissection }\end{array}\end{array}$ & 3 & 123 & 64.2 \\
Total thyroidectomy via an incision across the neck and chest & 1 & 123 & 2.4 \\
\hline
\end{tabular}
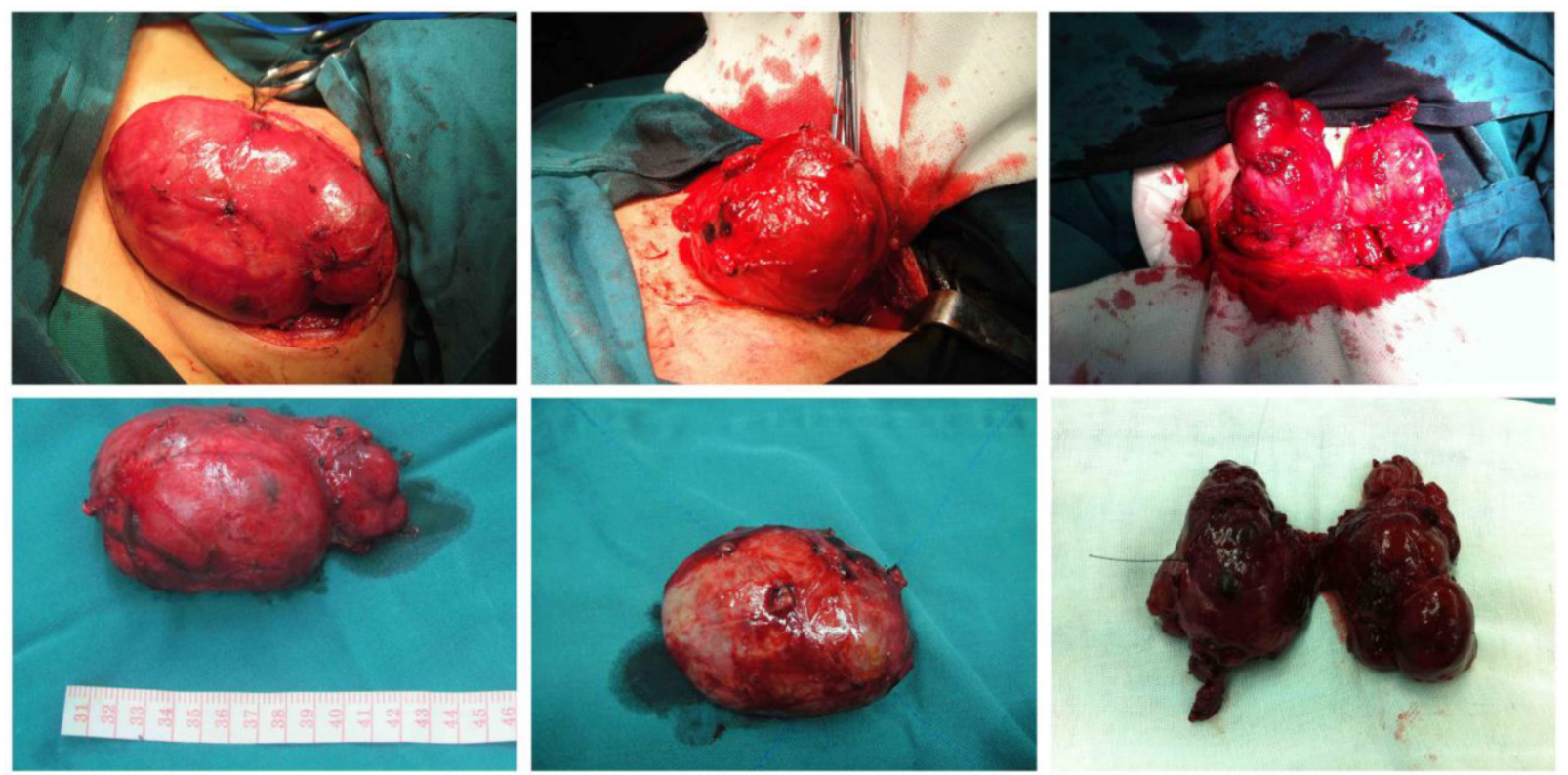

Figure 5 Curved incision in the neck. Intra-operative findings of giant nodular goiter. 

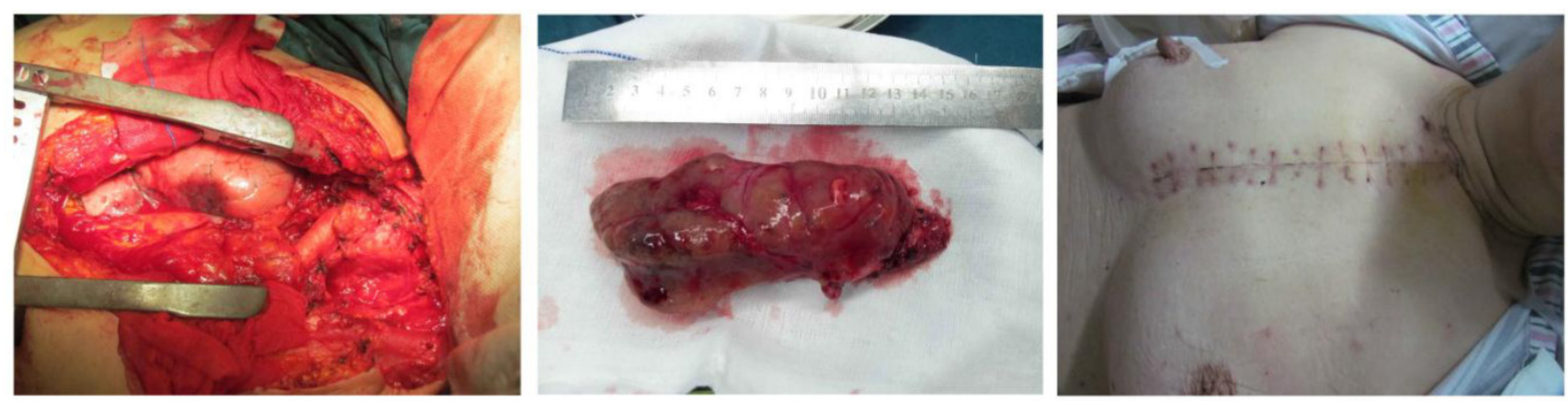

Figure 6 A T incision was made at the neck and chest: Intra-operative findings of giant nodular goiter at the posterior mediastinum.
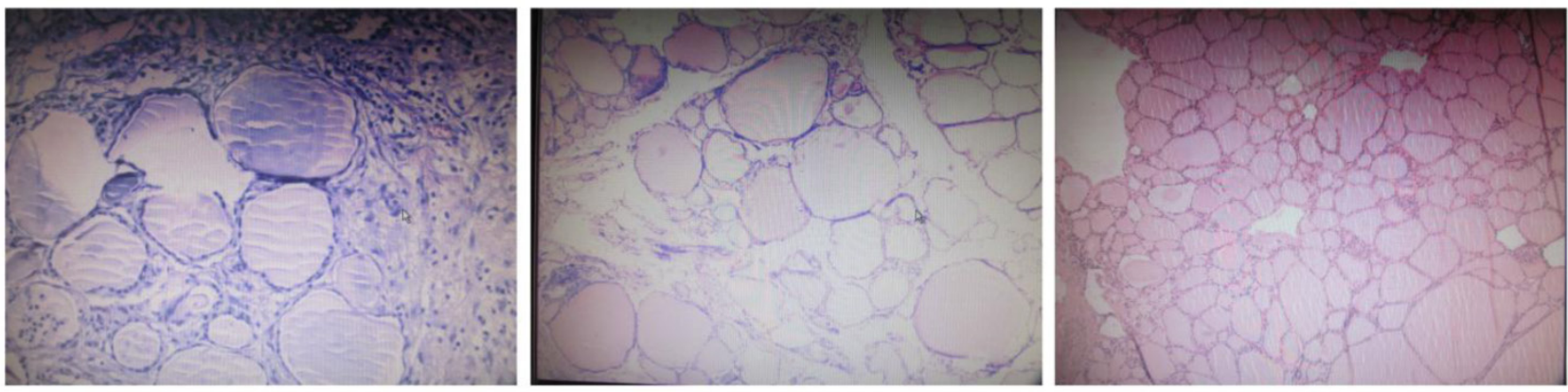

Figure 7 Histological examination of nodular goiter (HE staining; $\times 400)$. The follicular epithelial cells were flat and had small and oval nucleus. The cytoplasm was lightly stained and the follicular cavity was filled with colloid. In several follicule, increased blood vessels were noted accompanied by interlobular fibrous tissue hyperplasia.

\section{Post-operative follow up}

All these patients had no short term severe complications such as dyspnea or thyroid storm and other compilations are shown in Table 2. Hemorrhage was found in 3 patients who recovered after second surgery to treat bleeding points, and hoarseness occurred in 4 patients of whom 3 recovered within 1 week and 1 within 3 months. Five patients had transient hypocalcemia and recovered within 1 month after calcium supplement. Intra-operative tracheotomy was done in 2 patients due to tracheomalacia and extubation was done within 20 days after surgery but infection, subcutaneous emphysema, tracheal stenosis and dyspnea were not found in these 2 patients. Post-operative follow up was done for 9 months to 6 years and recurrence was not observed. Thyroxine or euthyrox (levothyroxine sodium) was administered in these patients for $3 \sim 6$ months aiming to maintain the upper limit of normal of T3 and T4. Hypersensitive hTSH was maintained at $0.1-0.5 \mathrm{uIU} / \mathrm{ml}$ to prevent or reduce recurrence.
Table 2. Number and percentage of postoperative complications

\begin{tabular}{llll}
\hline $\begin{array}{l}\text { Postoperative } \\
\text { complications }\end{array}$ & $\begin{array}{l}\text { Number of } \\
\text { patients }\end{array}$ & $\begin{array}{l}\text { Total number } \\
\text { of patients }\end{array}$ & $\begin{array}{l}\text { The percentage } \\
(\%)\end{array}$ \\
\hline Bleeding & 3 & 123 & 2.4 \\
Dyspnea & 0 & 123 & 0 \\
Thyroid crisis & 0 & 123 & 0 \\
hoarseness & 4 & 123 & 3.3 \\
Hypocalcemia & 1 & 123 & 0.8 \\
Tracheal incision & 2 & 123 & 1.6 \\
\hline
\end{tabular}

\section{Discussion}

\section{Indications for surgery}

Nodular goiter is usually multinodular lesion and surgical intervention is the major strategy for its treatment. The indications for nodular goiter include 1) bilateral nodular goiter compresses the transchea and dyspnea is present in the supine position; 2) multinodular goiter is accompanied by hyperthyroidism which is non-responsive to pharmacotherapy; 3) The nodules grow rapidly and have the risk for cancera- 
tion; 4) The nodular goiter is giant and affect the beauty. The nodular goiter is usually accompanied by thyroid carcinoma which is difficult to determine before surgery. Thus, for patients with evident nodules in the thyroid, surgical intervention may be considered to exclude the possibility of thyroid carcinoma.

\section{Surgical procedures and recurrence}

Studies revealed the recurrence rate of nodular goiter was $10 \sim 50 \%$ after surgery in other countries and $11.3 \%$ in China [10-12]. The nodular goiter has high incidence of recurrence and has the risk for canceration. For patients with recurrence, a second surgery may cause some complications such as damage to the parathyroid gland and recurrent laryngeal nerve. There is evidence showing that the incidence of complications was about $8 \%$ after surgery due to recurrence of nodular goiter. Among these complications, the incidence of damage to the recurrent laryngeal nerve was $0 \sim 14.0 \%$ and that of hypocalcemia was $1.2 \sim 10.6 \%$ [13-14]. Thus, early surgical intervention is recommended and total or subtotal thyroidectomy is preferred [15]. Currently, there is still controversy in the selection of surgical methods. The total and subtotal thyroidectomy is the major method and subtotal thyroidectomy has been a standard method in the treatment of nodular goiter [16]. Studies also demonstrated that total or subtotal thyroidectomy and neartotal thyroidectomy had no difference in the post-operative complications, and both surgical interventions were comparable in the subsequent treatment [17]. In addition, there is evidence showing that the age of $<40$ years and multiple nodules are the risk factors of recurrence after subtotal thyroidectomy, and thus total thyroidectomy is recommended for patients with bilateral multinodular goiter aiming to prevent a second surgery due to recurrence[14]. For patients with goiter in the posterior mediastinum, an incision is usually made in the neck and chest followed by total thyroidectomy [18].

\section{Pre-operative and intra-operative cautions}

Pre-operative preparation: The goiter is giant and rich in blood supply, the blood vessels and nerves are compress to dislocate and the mass has poor movement, which adds difficulty to the surgery. Thus, comprehensive pre-operative preparation is essential and includes: 1) red blood cell suspension (400 1200 ml) should be prepared on the basis of tumor size; 2) Routine detections of heart function, lung function, kidney function and coagulation. Once abnormality is present, corresponding treatment should be performed to return them to a nearly normal level; 3) Neck $X$ ray and CT are recommended to identify the compression of trachea and esophagus; 4) laryngoscopy is recommded to determine the location and movement of vocal cords; 5) For patients with hyperthyroidism, routine pre-operative preparation is essential (such as administration of methimazole, iodine and propranolol), the heart rate should be maintained at $<90$ beats/min and BMR at $<20 \sim 30 \%$. Once the primary cardiovascular diseases and respiratory diseases are excluded on the basis of medical history and related examinations, the increase in respiration and heart rate should be considered as a result of hyperthyroidism. Pre-operative administration of iodine preparations is pivotal. For patients with concomitant hyperthyroidism, surgery is not performed before the symptoms resolve or the thyroid storm might be present and is usually life-threatening.

\section{Selection of anesthetic methods}

General anesthesia following tracheal intubation and cervical plexus block can be used for thyroidectomy. When the mass is giant and cause tracheal stenosis or the patients present dyspnea before surgery, general anesthesia following tracheal intubation is recommended. In the present study, general anesthesia was done in 106 patients. When the tracheal cavity is normal and dyspnea is absent, cervical plexus block may be applied. In our study, cervical plexus block was done in 17 patients.

\section{Patient's position and surgical incision}

In the surgery, hyperextension of the neck is required (the shoulder is elevated to assure the head hypsokinesis) to completely expose the surgery field. The surgical incision is selected in the principle of complete exposure of thyroid. A curved incision (collar-shaped incision) is usually used and the both ends of the incision are slightly elevated. For patients with the lower part of the thyroid extending to the retrosternal region, the incision is slightly lower. For patients with giant goiter, an " $\mathrm{L}$ "-shaped incision is recommended.

\section{Protection of cervical major vessels}

During the surgery, the location of the common carotid artery should be kept in mind aiming to determine the location of internal jugular vein, which aimed to avoid the damage to the internal jugular vein. Before separation and dissection of the affected thyroid, the major vessels should be pushed away and blind clamping for hemostasis should be avoided.

\section{Management of vessels in the thyroid}

The giant goiter is rich in blood vessels the location of which is usually altered under this condition. 
On the thyroid, the veins and arteries are giant and have some branches. The thyroid usually connects to the major vessels in the neck. Improper management has the risk for massive hemorrhage. The upper part of the thyroid is rich in blood vessels which are often ligated with 7-0 suture. These blood vessels were clamped at $2 \mathrm{~mm}$ away the upper and lower parts of the thyroid followed by disconnection of blood vessels which are then ligated independently for prevention against massive hemorrhage. When there is no space between the upper part of the thyroid and the mandibular angle, management of the lower part and the isthmus of the thyroid is preferred and then the side of the thyroid is managed. For the inferior thyroid artery, the liagtion of its branches should be done on the thyroid and the major artery should not be ligated, which not only assures the hemostasis, but has no risk for damage to the parathyroid and recurrent laryngeal nerve. Then, the thyroid is retracted downward aiming to increase the space between the upper part of the thyroid and the mandibular angle. Then, the superior thyroid artery is ligated twice. In modern medicine, more and more surgeons advocate ultrasound knife or bipolar fusion to stop bleeding for the advantages such as excellent effects and time-saving. The head of ultrasound knife is coarse and large and, therefore, more suitable for thyroid lobectomy or coarse vessel cutting. The ultrasound knife is associated with a higher risk of nerve injuries in cutting blood vessels in the superior and inferior thyroid than the conventional silk suture ligation. Bipolar electrode is thin and produces little heat and, therefore, can be used to stop bleeding of tiny vessels and around nerves. Proper combination of ultrasound knife, bipolar fusion and conventional silk sutures can produce better hemostatic effects.

In the present study, the giant goiter was found to be adherent to the surrounding tissues in 9 patients. The improper retraction or blunt separation led to excessive bleeding. After compression for hemostasis and blood transfusion, the experienced surgeons were employed and subtotal thyroidectomy was done. Hemostasis was achieved. In 3 patients, post-operative hemorrhage was present. The wound was then exposed and the bleeding site was identified followed by hemostasis. Thus, severe complication was not found.

\section{Protection of recurrent laryngeal nerve}

In the surgery for giant goiter, hoarseness is easily induced due to the limited operation space and large anatomical variation of recurrent laryngeal nerve. In this study, 4 patients developed hoarseness post surgery. Of the 4 patients, 3 recovered within 1 week and 1 recovered within 3 months. For the latter patient, the recurrent laryngeal nerve and the tumor were adhered and the recurrent laryngeal nerve was injured in separating the 2 organs. Transient hoarseness has a favorable prognosis. It generally occurs within 1 to 3 days post surgery and disappears in 1 week post surgery. This nerve is injured possibly by: 1) nerve edema from over stretching during surgery; 2) tissue edema post surgery or compression by secondary small hematoma; and 3) compression by constriction of fibrous scar. Some scholars deem that exposing the recurrent laryngeal nerve is the effective measure to prevent injury of this nerve in subtotal thyroidectomy. The author considers that this nerve is easily injured in benign giant goiter and should be routinely exposed in second surgery. Additionally, in developed countries, intraoperative nerve monitoring has been applied for more than 10 years in clinical practice and gradually develops into a comprehensive multidisciplinary monitoring system. The intraoperative monitoring technique is an important component in the field of modern surgery. It uses the combination of electromyogram and tracheal cannula to monitor motor nerves during surgery. In this technique, surface electromyogram electrodes and the endotracheal tube are placed in the medial vocal cord. This procedure is more reasonable and safer and greatly reduces medical disputes. Therefore, the American Association of Endocrine Surgeons (AAES) generalizes the recurrent laryngeal nerve monitor during surgery as the guide.

\section{Protection of parathyroid}

For patients with giant goiter, hypoparathyroidism is common after surgery, which is usually attributed to the mis-dissection or damage to the parathyroid or the impairment of blood supply [19]. The parathyroid locates at the back of thyroid. The upper parathyroid has fixed location and is often identified at the back of upper thyroid and close to the larynx cartilage. The location of lower parathyroid is not fixed and various and usually found at the back of lower thyroid. The protection of parathyroid is required the preservation of the capsule at the back of thyroid. Thus, the inferior thyroid artery is not ligated aiming to preserve the capsule at the back. The loose tissues at the lower part of thyroid should be preserved. The dissected samples should be double-checked. Once the parathyroid is found, the parathyroid at $1 \mathrm{~mm} \times 1 \mathrm{~mm}$ should be collected and implanted in the sternocleidomastoid [20-21].

\section{Management of concomitant thyroid cancer}

The giant goiter has the risk for canceration. 
When the thyroid is adherent to the muscle or surrounding major blood vessels, frozen pathology is required. Once the lesions invade the carotid artery/vein and trachea, palliative resection is required, which may extend the survival time for $3 \sim 4$ years. Radical thyroidectomy may extend the survival time for 10 15 years. Thus, if possible, radical thyroidectomy should be performed. In the present study, 3 patients with concomitant papillary thyroid carcinoma, the total thyroidectomy of affected side, total dissection of isthmus and subtotal thyroidectomy of contralateral thyroid were performed accompanied by lymph node dissection.

\section{Management of retrosternal goiter}

The retrosternal goiter refers to the goiter the lower part of which can not be palpated or the goiter extending to $3 \mathrm{~cm}$ downward the sternum vein notch. The principles for the treatment of retrosternal goiter are as follows: 1) The lower part of the thyroid is retracted out of the retrosternal region with a suture accompanied by blunt separation; 2) Once the thyroid can not be taken out, forced separation is forbidden or the pleura may be damaged resulting in pneumothorax, or the innominate vein and inferior thyroid vein may be damage resulting in massive hemorrhage. Under this condition, the upper part of the thyroid can be dissected first to expand the surgical field and then the lower part of the thyroid is retracted upward accompanied by blunt separation. In addition, when the residual thyroid is adherent to the innominate vein, the lower part of the thyroid can not be exposed and the blood vessels at the lower part are unlikely to be ligated, the sternum should be separated to expose the lower part of the thyroid aiming to avoid the damage to the innominate vein [22-25].

\section{Competing Interests}

The authors have declared that no competing interest exists.

\section{References}

[1] Yeung MJ, Serpell JW. Management of the solitary thyroid nodule. Oncologist 2008;13: 105-112.

[2] Tae HJ, Lim DJ, Baek KH, et al. Diagnostic value of ultrasonography to distinguish between benign and malignant lesions in the management of thyroid nodules. Thyroid 2007; 17: 461-466.

[3] Ríos Zambudio A, Rodríguez González JM, Galindo Fernández PJ, et al. Clinical recurrence of multinodular goiter after surgery. A multivariate study on the risk factors. Rev Clin Esp 2005; 205: 9-13.

[4] Blanco Carrera C, García-Díaz JD, Maqueda Villaizán E, et al. Diagnostic efficacy of fine needle aspiration biopsy in patients with thyroid nodular disease. Analysis of 510 cases. Rev Clin Esp 2005;205: 374-378.

[5] Bhagat MC, Dhaliwal SS, Bonnema SJ, Hegedüs L, Walsh JP: Differences between endocrine surgeons and endocrinologists in the management of non-toxic multinodular goitre. Br J Surg 2003; 90: 1103-1112.

[6] Wesche MF, Tiel-V Buul MM, Lips P, Smits NJ, Wiersinga WM. A randomized trial comparing levothyroxine with radioactiveiodine in the treatment of sporadic nontoxic goiter. J Clin Endocrinol Metab 2001; 86: 998-1005.

[7] Huysmans D, Hermus A, Edelbroek M, Barentsz J, Corstens F, Kloppenborg P. Radioiodine for non-toxic multinodular goiter. Thyroid 1997; 7: 235-239.

[8] Gharib H, Papini E, Paschke R, Duick DS, Valcavi R, Hegedu“s L, Vitti P. American Association of Clinical Endocrinologists, Associazione Medici Endocrinologi, and European Thyroid Association medical guidelines for clinical practice for the diagnosis and management of thyroid nodules: Executive Summary of Recom-mendations. J Endocrinol Invest 2010; 33:287-291.

[9] Cooper DS, Doherty GM, Haugen BR, Hauger BR, Kloos RT, Lee SL, Mandel SJ, Mazzaferri EL, McIver B, Pacini F, Schlumberger M, Sherman SI, Steward DL, Tuttle RM. Revised American Thy-roid Association management guidelines for patients with thyroidnodules and differentiated thyroid cancer. Thyroid 2009; 19: 1167-1214.

[10] Tezelman S, Borucu I, Senyurek Giles Y, et al. The change in surgical practice from subtotal to near-total or total thyroidectomy in the treatment of patients with benign multinodular goiter. World J Surg 2009; 33: 400-405.

[11] Agarwal G, Aggarwal V. Is total thyroidectomy the surgical procedure of choice for benign multinodular goiter? An evidence-based review. World J Surg 2008; 32: 1313-1324.

[12] Xu G, Li Z, Chen F. recurrence factors after Nodular goiter surgery. Chin Gen Surg 2002; 17: 432-433.

[13] Mishra A, Agarwal A, Agarwal G, et al. Total thyroidectomy for benign thyroid disorders in an endemic region. World J Surg 2001; 25: 307-310.

[14] Gibelin H, Sierra M, Mothes D, et al. Risk factors for recurrent nodular goiter after thyroidectomy for benign disease: case-control study of 244 patients. World J Surg 2004;28: 1079-1082.

[15] Friguglietti CU, Lin CS, Kulcsar MA. Total thyroidectomy for benign thyroid disease. Laryngoscope 2003; 113:1820-1826.

[16] Unalp HR, Erbil Y, Akguner T, et al. Does near total thyroidectomy offer advantage over total thyroidectomy in terms of postoperative hypocalcemia? Int J Surg 2009; 7: 120-125.

[17] Vaiman M, Nagibin A, Hagag P, et al. Subtotal and near total versus total thyroidectomy for the management of multinodular goiter. World J Surg 2008; 32: 1546-1551.

[18] Chong CF, Cheah WK, Sin FL, et al. Posteriorm ediast inal goiter. Asian Cardiovasc Thorac Ann 2004; 12:263-265.

[19] Rios Zambudio A, Rodriguez Gonzalez JM, Galindo Fernandez PJ, et al . Clinical recurrence of multinodular goiter after surgery. A multivariate study on the risk factors. Rev Clin Esp 2005; 205: 9.

[20] Singh B, Lucente FE, Shaha AR. Substernal goiter: a clinical review. Am J Otolaryngol 1994; 15: 409-416.

[21] Torre G, Borgonovo G, Amato A, et al. Surgical management of substernal goiter: analysis of 237 patients. Am Surg 1995; 61: 826-831.

[22] WhiteML, Doherty GM, Gauger PG. Evidence-based surgical management of substernal goiter. World J Surg 2008; 32: 1285-1300.

[23] Rosato L, Avenia N, Bernante P, De PalmaM, Gulino G, Nasi PG, Pelizzo MR, Pezzullo L. Complications of thyroid surgery: analysis of a multicentric study on 14,934 patients operated on inItaly over 5 years. World J Surg 2004; 28:271-276.

[24] Agha A, Glockzin G, Ghali N, Iesalnieks I, Schlitt HJ. Surgical treatment of substernal goiter: an analysis of 59 patients. Surg Today 2008;38:505-511.

[25] Wang LS. Surgical management of a substernal goiter. Formosan J Surg 2012;45: 41-44. 